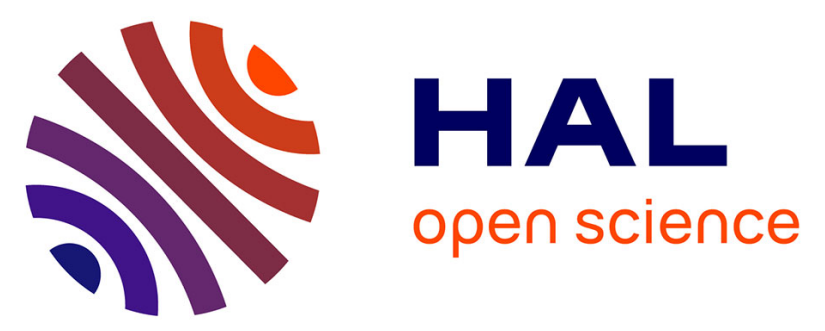

\title{
Electronic properties of embedded graphene: doped amorphous silicon/CVD graphene heterostructures
}

Hakim Arezki, Mohamed Boutchich, David Alamarguy, Ali Madouri, José Alvarez, Pere Roca I Cabarrocas, Jean-Paul Kleider, Fei Yao, Young Hee Lee

\section{- To cite this version:}

Hakim Arezki, Mohamed Boutchich, David Alamarguy, Ali Madouri, José Alvarez, et al.. Electronic properties of embedded graphene: doped amorphous silicon/CVD graphene heterostructures. Journal of Physics: Condensed Matter, 2016, 28 (40), pp.404001. 10.1088/0953-8984/28/40/404001 . hal01363551

\section{HAL Id: hal-01363551 \\ https://hal.science/hal-01363551}

Submitted on 8 Nov 2016

HAL is a multi-disciplinary open access archive for the deposit and dissemination of scientific research documents, whether they are published or not. The documents may come from teaching and research institutions in France or abroad, or from public or private research centers.
L'archive ouverte pluridisciplinaire HAL, est destinée au dépôt et à la diffusion de documents scientifiques de niveau recherche, publiés ou non, émanant des établissements d'enseignement et de recherche français ou étrangers, des laboratoires publics ou privés. 


\title{
Electronic properties of embedded graphene: doped amorphous silicon/CVD graphene heterostructures
}

\author{
Hakim Arezki ${ }^{1}$, Mohamed Boutchich ${ }^{1}$, David Alamarguy ${ }^{1}$, Ali Madouri ${ }^{2}$, José Alvarez ${ }^{1}$, Pere Roca i Cabarrocas ${ }^{3}$, \\ Jean-Paul Kleider ${ }^{1}$, Fei Yao ${ }^{4}$, and Young Hee Lee ${ }^{4,5}$ \\ ${ }^{1}$ GeePs, CNRS UMR8507, Centralesupelec, Univ Paris-Sud, Sorbonne Universités-UPMC Univ Paris 06, 11 rue \\ Joliot-Curie, Plateau de Moulon, 91192 Gif-sur-Yvette Cedex, France. \\ ${ }^{2}$ LPN-CNRS, Route de Nozay, 91460 Marcoussis, France. \\ ${ }^{3}$ LPICM, CNRS, Ecole Polytechnique, Université Paris-Saclay, 91128 Palaiseau, France \\ ${ }^{4}$ Center for Integrated Nanostructure Physics, Institute for Basic Science, Sungkyunkwan University, Suwon 440- \\ 747, Korea \\ ${ }^{5}$ Department of Energy Science and Department of Physics, Sungkyunkwan University, Suwon 440-747, Korea. \\ Email: mohamed.boutchich@upmc.fr
}

Large-area graphene film is of great interest for a wide spectrum of electronic applications, such as field effect devices, displays, and solar cells, among many others. Here, we fabricated heterostructures composed of graphene (Gr) grown by chemical vapor deposition (CVD) on copper substrate and transferred to $\mathrm{SiO}_{2} / \mathrm{Si}$ substrates, capped by n or p-type doped amorphous silicon (a-Si:H) deposited by plasma-enhanced chemical vapor deposition. Using Raman scattering we show that despite the mechanical strain induced by the a-Si:H deposition, the structural integrity of the graphene is preserved. Moreover, Hall effect measurements directly on the embedded graphene show that the electronic properties of CVD graphene can be modulated according to the doping type of the a-Si:H as well as its phase i.e. amorphous or nanocrystalline. The sheet resistance varies from $360 \Omega /$ sq to $1260 \Omega /$ sq for the (p)-a$\mathrm{Si}: \mathrm{H} / \mathrm{Gr}$ (n)-a-Si:H/Gr, respectively. We observed a temperature independent hole mobility of up to $1400 \mathrm{~cm}^{2} / \mathrm{Vs}$ indicating that charge impurity is the principal mechanism limiting the transport in this heterostructure. We have demonstrated that embedding CVD graphene under a-Si:H is a viable route for large scale graphene based solar cells or displays applications.

\section{Introduction}

The recent advances in large-scale synthesis of graphene and alternative 2D materials [1-3] offer new opportunities to investigate various heterostructures composing the building blocks of new devices and architectures [4,5]. However, the electronic properties of graphene differ according to the growth technology utilized [6], the grain size, the interaction with the underlying substrate as well as the non-intended doping that systematically occurs during fabrication [7]. In addition, graphene and 2D materials are extremely sensitive to environment, implying uncontrollable variations such as the threshold voltage of 2D based field effect devices [8,9]. On the other hand, the modulation of the electronic properties of graphene is a key prerequisite to envision its integration as an active 
material but also as an electrode [10]. In that regard, a lot of work has been devoted to the study of more or less stable organic or inorganic materials deposited onto graphene [11-13], [14], [15], [16]. Field effect doping and dopant incorporation at these interfaces allow the modulation of the work function, the carrier density and sheet resistance as well as the mobility [17-21], [22], [23]. Kim et al. [21] demonstrated GaN-based light-emitting diodes where the conductivity of graphene was substantially improved when capped with silicon nitride (SiNx) but did not discuss the electrostatic impact of the fixed charges trapped in the SiNx. Gluba et al. [24] studied CVD graphene capped with a-Si:H and polycrystalline silicon. Coupling Raman spectroscopy and Hall effect measurements, they observed that the structural features of graphene where preserved and the mobility is limited at room temperature due to phonon scattering [25-27], [28], [29] . They concluded that large-scale CVD graphene applications might be hindered due this latter effect. Recently, Ho et al. [30] demonstrated a graphene/TiOx photoactive heterostructure cathode to fabricate an (n) graphene/(p) Si Schottky junction with power conversion efficiency $>10 \%$. These recent advances [31] shine light on the integration of graphene in solar cell applications that are already competitive with respect to organic based demonstrators but also on field effect devices where graphene can be implemented as a channel or a floating gate [3,32].

Here, we investigate graphene/doped amorphous silicon heterostructures. The chemical elements present on the graphene were determined by X-ray spectroscopy (XPS) and the work function was measured by ultraviolet photoemission spectroscopy (UPS). The homogeneity of the transferred graphene layers was confirmed by Raman spectroscopy. The latter also showed that the mechanical strain hinders the field effect doping resulting from the fixed charges in the capping a-Si:H. Hall effect measurements as a function of temperature show that the doping nature of the a-Si:H influences the electronic properties such as the sheet resistance that drops down to $430 \Omega / s q$ for the (p) a-Si:H/Gr for a hole mobility of $1400 \mathrm{~cm}^{2} / \mathrm{Vs}$. Moreover, our measurements do not present any temperature dependence, that is in a sharp contrast with the work of Gluba et al. [24] on undoped a-Si:H. At last we have shown that the recrystallization of the doped a-Si:H film relaxes the mechanical strain [33], [34] and only the field effect doping remains. We have demonstrated that embedding CVD graphene under a-Si:H is a route for large scale graphene based solar cells or display applications.

\section{Heterostructures and experiments}

Graphene layers were grown on commercially available $100 \mu \mathrm{m}$ thick copper foil (from Nilaco, 99.96\%) annealed at $1075{ }^{\circ} \mathrm{C}$ with $1000 \mathrm{sccm}$ Ar and $500 \mathrm{sccm} \mathrm{H}_{2}$ for $2 \mathrm{~h}$ by CVD at atmospheric pressure. The annealed copper foil was polished using the chemical mechanical polishing method (6 ml copper etchant was diluted in $100 \mathrm{ml}$ water). The polished $\mathrm{Cu}$ film was placed in a 2 inch quartz tube chamber and heated up to $1075{ }^{\circ} \mathrm{C}$ with $1000 \mathrm{sccm} \mathrm{Ar}$ and 500 sccm $\mathrm{H}_{2}$ for annealing for $2 \mathrm{~h}$ to remove residuals on the copper surface [35,36]. During the atmospheric CVD growth, the $\mathrm{H}_{2}$ gas was reduced to $170 \mathrm{sccm}$ and $200 \mathrm{sccm}$ of $\mathrm{CH}_{4}$ (0.1\% diluted in Ar was injected) for 25 min. After growth, the $\mathrm{CH}_{4}$ was turned off and the chamber was cooled down to room temperature. The copper foil was subsequently etched in $\mathrm{FeCl}_{3}$ etchant and the graphene was transferred onto $\mathrm{SiO}_{2} / \mathrm{Si}$ substrate using PMMA resist. To fabricate the heterostructure, p-type and n-type a-Si:H films were deposited by standard plasma-enhanced chemical vapor deposition at $175^{\circ} \mathrm{C}$ using a multiplasma-monochamber reactor [37], from the dissociation of silane 
mixed with trimethyl-boron or phosphine. The oxygen content of the films, as deduced from secondary ion mass spectrometry measurements is $\sim 2 \times 10^{19} \mathrm{~cm}^{-3}$ resulting from the residual impurities in the reactor [38].

We have studied both configurations : i) the graphene on top of a-Si:H and graphene coated by the a-Si:H thin films which have the advantage of providing a encapsulating layer making the measurements less sensitive to the atmosphere conditions.

We have investigated the electronic properties of graphene based heterostructures: (i) graphene on top of doped a$\mathrm{Si}: \mathrm{H}$ on silicon oxide and (ii) graphene on $\mathrm{SiO}_{2}$ capped with $10 \mathrm{~nm}$ doped a-Si:H, Figure 1. The Raman measurements were performed with a frequency-doubled NdYag laser operating at $532 \mathrm{~nm}$ as the excitation source with up to $20 \mathrm{~mW}$ continuous wave output power. High laser power can induce crystallization of the a-Si:H, so the laser power was judiciously tuned to investigate the Raman signature before and after crystallization of the a-Si:H. XPS and UPS measurements for the study of the chemical composition and the work function (WF), respectively, have been performed using a PHI 5000 Versaprobe spectrometer (Physical Electronics) operating at a base pressure of $10^{-9} \mathrm{mbar}$. For the XPS analysis, a focused monochromatic Al $\mathrm{K}_{\alpha} \mathrm{X}$-ray source (hv $=1486.6 \mathrm{eV}$ ) was used. The work function was determined from the secondary electron cut-off in UPS experiments using a He I discharge lamp $(\mathrm{h} v=21.2 \mathrm{eV}$ ). The position of the Fermi level was calibrated by measuring the Fermi edge of a sputtered gold sample. During the WF measurements, a bias of $-8 \mathrm{~V}$ was applied to the sample. Pieces of the doped a-Si:H/Gr heterostructures have been patterned in Hall bars using optical lithography to investigate the transport properties of the encapsulated graphene, Figure 1. These measurements were performed in an ambient environment.

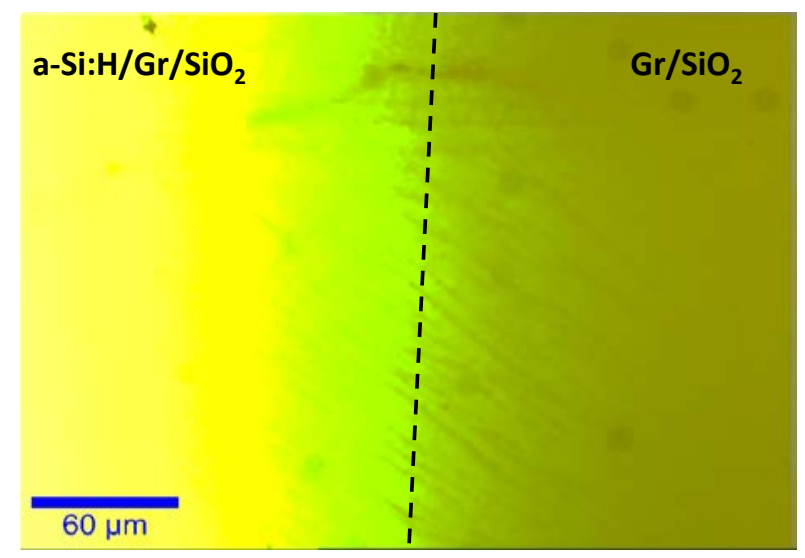

Figure 1: Optical image of the graphene sample utilized in the experiments. The yellow region corresponds to the graphene capped with a-Si:H whereas the green region corresponds to graphene on $\mathrm{SiO}_{2}$. The light green region corresponds to a thinner thickness of a-Si:H that diffused under the hard mask during the deposition.

\section{Results and discussion}

Let us first address the case where the graphene films are deposited on top of the a-Si:H layers. Figure 2 presents the Raman spectra of CVD graphene transferred onto $10 \mathrm{~nm} \mathrm{n-} \mathrm{and} \mathrm{p-type} \mathrm{a-Si:H.} \mathrm{All} \mathrm{samples} \mathrm{show} \mathrm{a} \mathrm{peak} \mathrm{at} 480 \mathrm{~cm}^{-1}$, characteristic of a-Si:H, as well as the G and 2D peaks of graphene. However, the G peak shift is $7 \mathrm{~cm}^{-1}$ and the 2D peak shift is $3 \mathrm{~cm}^{-1}$ on the p-type a-Si:H with respect to the n-type a-Si:H. This indicates that the graphene on p-type 
a-Si:H has been slightly n-type doped. This doping may result from contamination subsequent to the transfer process or possibly from a charge transfer at the heterostructure interface, Figure 2.

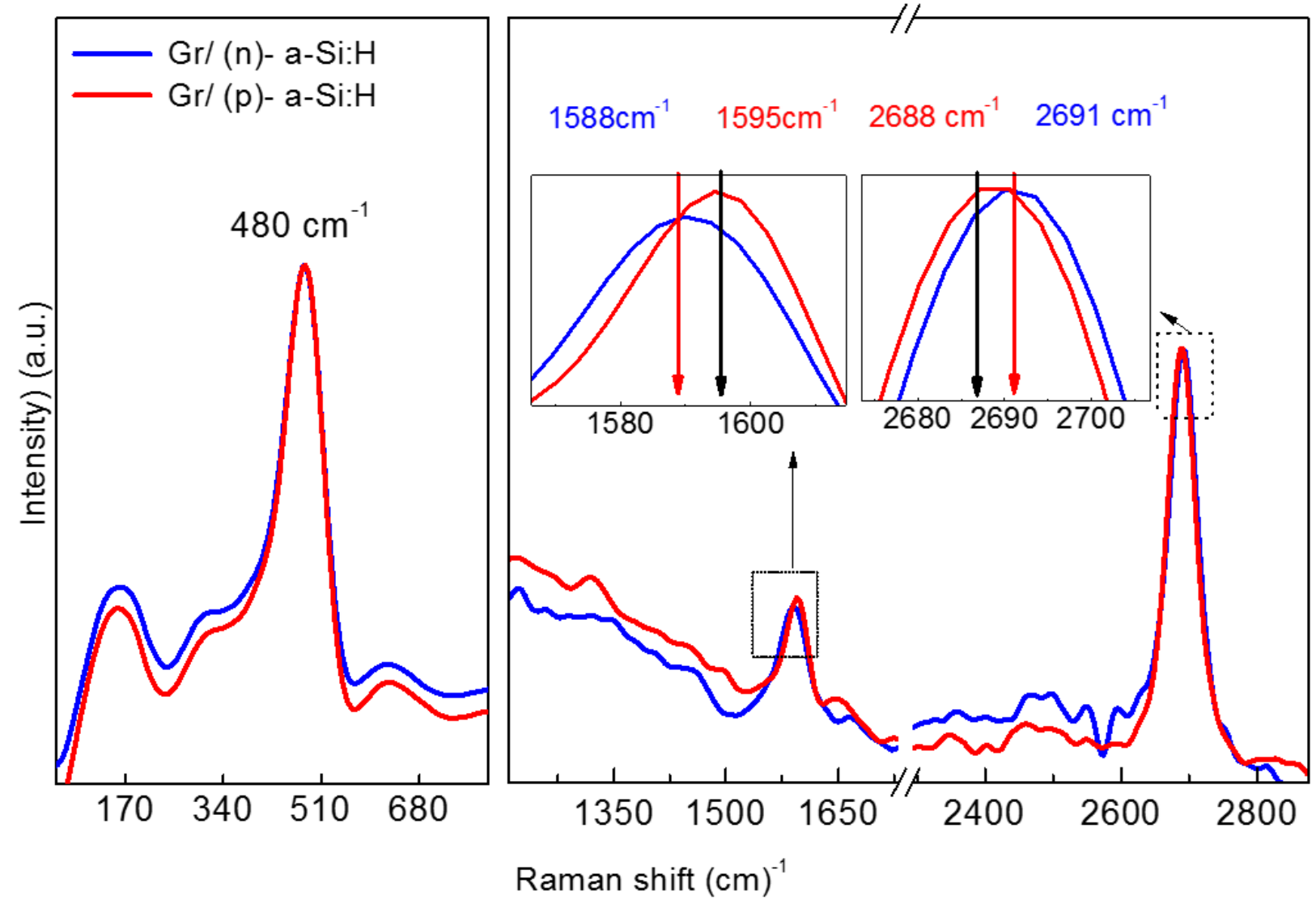

Figure 2: Raman spectra of CVD graphene transferred onto n- and p-type a-Si:H.

Indeed, XPS measurements (not shown) showed the presence of oxygen $(\mathrm{O})$, fluorine $(\mathrm{F})$, nitrogen $(\mathrm{N})$ and chlorine (Cl) species on graphene. Oxygen is known as a p-type dopant whereas $\mathrm{F}$ and $\mathrm{N}$ are n-type dopants [39]. The concentrations of these species vary from sample to sample and they are not controllable. We have carried out transport measurements on graphene on top of the doped a-Si:H.

\begin{tabular}{cccc}
\hline \hline Heterostructures & $\mathrm{R}_{\mathrm{sh}}(\Omega / \mathrm{sq})$ & $\mathrm{n}_{\mathrm{s}}\left(\mathrm{cm}^{2}\right)$ & $\mu\left(\mathrm{cm}-{ }^{2} / \mathrm{Vs}\right)$ \\
\hline \hline $\mathrm{Gr} / \mathrm{SiO}_{2}$ & 450 & $1 \times 10^{13}$ & 1400 \\
$\mathrm{Gr} /(\mathrm{n})-\mathrm{a}-\mathrm{Si}: \mathrm{H} / \mathrm{SiO}_{2}$ & 420 & $8 \times 10^{12}$ & 1800 \\
$\mathrm{Gr} /(\mathrm{p})-\mathrm{a}-\mathrm{Si}: \mathrm{H} / \mathrm{SiO}_{2}$ & 780 & $4 \times 10^{12}$ & 2000 \\
\hline \hline
\end{tabular}

Table 1: Transport properties for graphene transferred onto amorphous silicon/silicon oxide stacks. These data represent average between $1.3 \mathrm{~K}$ and $300 \mathrm{~K}$.

Table 1 shows the temperature dependence of the Hall-effect mobility, charge carrier density, and sheet resistance of transferred CVD graphene (Gr) on top of $\mathrm{SiO}_{2}$, as well as on (p)-a-Si:H/ $\mathrm{SiO}_{2}$ and (n)-a-Si:H/SiO 2 stacks. We do not observe any dominant scattering mechanism and the as-transferred layers exhibit hole conduction with electrical properties that are nearly temperature independent. Note that the sheet resistance of graphene on both types of aSi:H films presents values that become interesting for transparent electrode application without any additional 
doping process. UPS measurements confirm that the heterostructures are electronically different. WF is equal to $4.35 \mathrm{eV}$ for the $\mathrm{Gr} /(\mathrm{p})-\mathrm{a}-\mathrm{Si}: \mathrm{H}$ heterostructure and $4.15 \mathrm{eV}$ for the $\mathrm{Gr} /(\mathrm{n})-\mathrm{a}-\mathrm{Si}: \mathrm{H}$ one. However, it is questionable to assign this $0.2 \mathrm{eV}$ difference to a charge transfer at the interface of the heterostructure. The carrier density is doubled between $\mathrm{Gr} /(\mathrm{p})-\mathrm{a}-\mathrm{Si}: \mathrm{H}$ and $\mathrm{Gr} /(\mathrm{n})-\mathrm{a}-\mathrm{Si}: \mathrm{H}$ while the mobility decreases from 2100 to $1800 \mathrm{~cm}^{2} / \mathrm{Vs}$. It is likely that this change is a consequence of the unintended doping during the transfer and fabrication processes.

In order to elucidate whether there could be charge transfer between a-Si:H and graphene, we have studied capped graphene i.e. (n)-a-Si:H/Gr and (p)-a-Si:H/Gr. Here, a $10 \mathrm{~nm}$ thick doped a-Si:H layer has been deposited onto Gr and Hall bars have been patterned. The graphene has been contacted first and the a-Si:H film has been etched to reach the contact pads. Figure 3 shows the Raman spectra of the different heterostructures benchmarked to the graphene onto $\mathrm{SiO}_{2}$. The measurements are carried out directly on different locations of the Hall bar device to take into account the homogeneity of the sample.

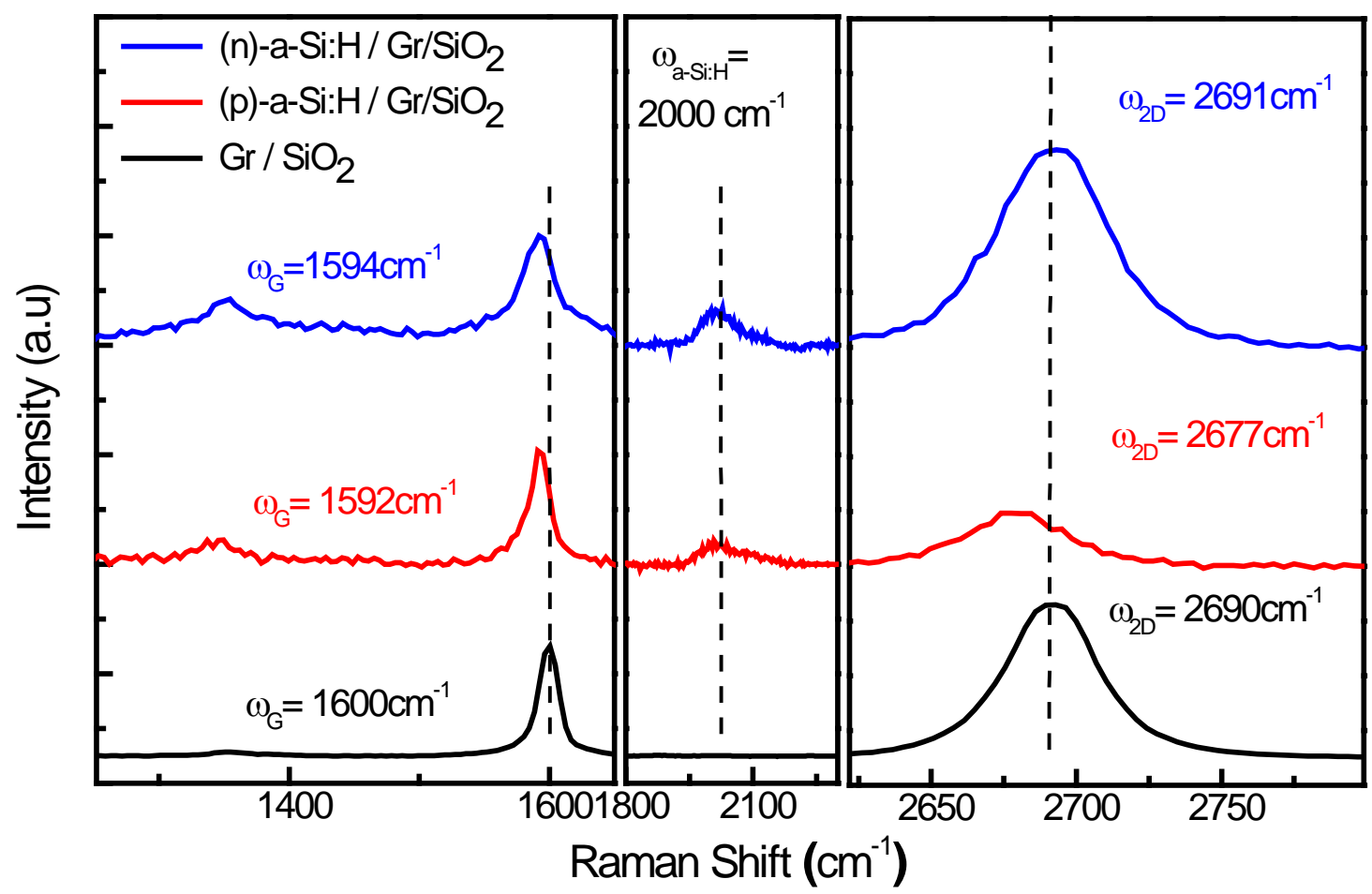

Figure 3: Raman spectra of the graphene on $\mathrm{SiO}_{2}$ substrate capped with doped a-Si:H. For clarity, the spectra are shifted vertically.

Interestingly, the D, G and 2D Raman modes of the graphene are preserved after deposition of a-Si:H indicating that the capping process is nondestructive although the low intensity of the D mode is slightly higher for the (n)-a-Si: $\mathrm{H} / \mathrm{Gr}$ compared to the (p)-a-Si:H/Gr heterostructure. However compared to the $\mathrm{Gr} / \mathrm{SiO}_{2}$ sample, the $\mathrm{G}$ and 2D modes of both a-Si:H based heterostructures exhibit a shift to lower frequencies, Figure 3. Moreover, for the (p)-a$\mathrm{Si}: \mathrm{H} / \mathrm{Gr}$ heterostructure, the 2D/G ratio decreases. This trend could result from mechanical strain induced by the boron or phosphorus doped a-Si:H layers [40,41], or from field effect doping from the charges at the interface. Although field effect doping cannot be excluded, it is clearly hindered by the mechanical strain that is the dominant 
mechanism as can be seen by the downshift observed on both the G and 2D modes for the two types of heterostructures [42,43].

We carried out Hall effect measurements as a function of temperature to evaluate the electronic properties of the capped graphene. Figure 4 shows the sheet resistance, mobility and carrier density of the embedded graphene benchmarked to graphene on $\mathrm{SiO}_{2}$.

a)

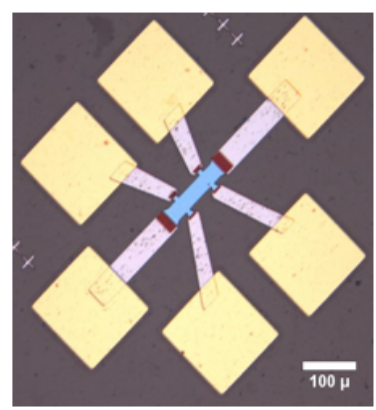

b)

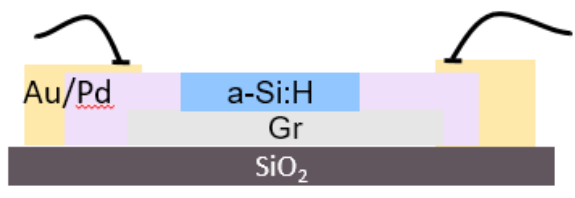

c)

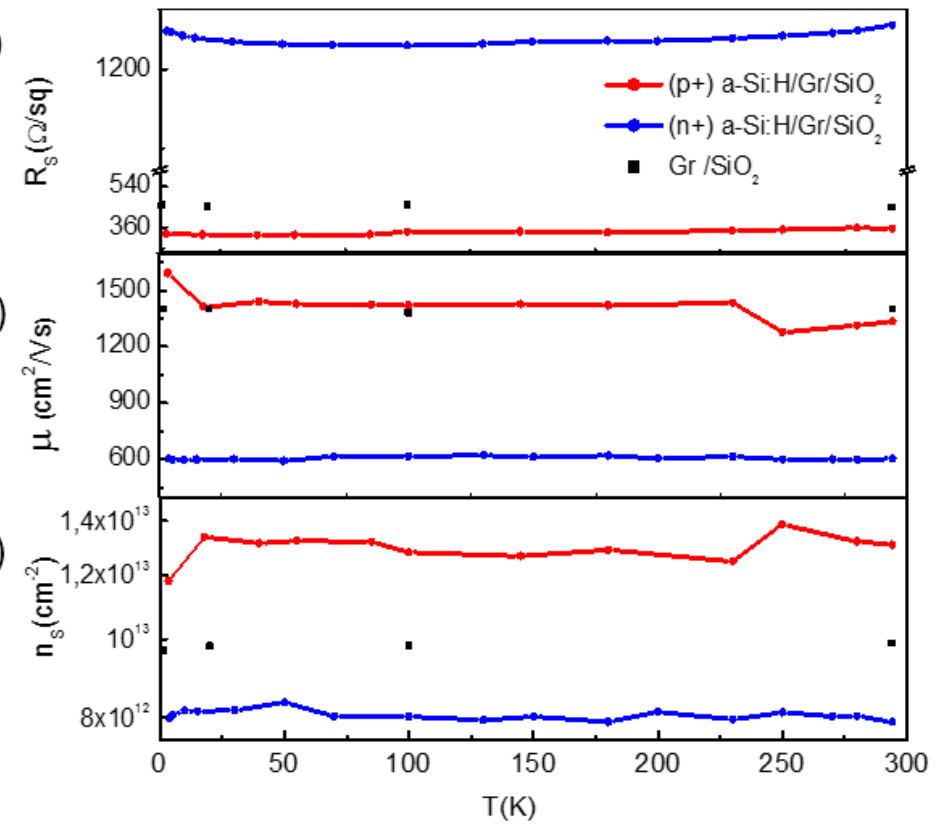

Figure 4: Graphene Hall bars capped with a-Si:H (a) optical image (30x70 $\mu \mathrm{m})$, (b) schematic of the Hall bars, temperature dependence of (c) sheet resistance, (d) Hall mobility and (e) charge carrier density of graphene capped with n or p-type a-Si:H. The dashed line represents data on $\mathrm{SiO}_{2}$.

Here both capped heterostructures i.e. (n,p) a-Si:H/Gr, present hole conduction with an average mobility of 1400 $\mathrm{cm}^{2} / \mathrm{Vs}$ and a sheet resistance as low as $420 \Omega / \mathrm{sq}$ for (p)-a-Si:H/Gr. For the latter the carrier density has increased to $1.3 \times 10^{13} \mathrm{~cm}^{-2}$ compared to the $9.8 \times 10^{12} \mathrm{~cm}^{-2}$ measured on $\mathrm{SiO}_{2}$ that is known to present hole accumulation. On the other hand, (n)-a-Si:H/Gr presents a lower mobility of $600 \mathrm{~cm}^{2} / \mathrm{Vs}$ with a sheet resistance of $1300 \Omega / \mathrm{sq}$ and a carrier density of $8 \times 10^{12} \mathrm{~cm}^{-2}$. The lower mobility observed for the (n)-a-Si:H/Gr may be a result of structural defects induced by the mechanical strain. Clearly, the doping type of the as-deposited a-Si:H influences the electronic properties through field effect doping induced by the fixed charges at the interfaces notably for the p-type sample. This doping has already been suggested by the Raman spectra of Figure 3; although the shift induced by the doping is hindered by the mechanical strain, the 2D mode intensity of the $\mathrm{Gr} /(\mathrm{p})$-a-Si:H reduces and the full width half maximum increases. Using the measured carrier density we can calculate the position of the Fermi level $\mathrm{E}_{\mathrm{F}}$ relative to the Dirac point $E_{D}: E_{D}-E_{F}=-0.33 \mathrm{eV}$ for (n)-a-Si:H/Gr and $E_{D}-E_{F}=0.65 \mathrm{eV}$ for (p)-a-Si:H/Gr. Note that we do not observe any temperature dependence that is in contrast with the work on graphene capped with undoped a-Si:H by Gluba et al. that observed a strong temperature dependence of capped graphene assigned to phonon assisted 
scattering starting at $100 \mathrm{~K}$ [24]. In our configuration, the graphene sheet is embedded between $\mathrm{SiO}_{2}$ substrate and doped a-Si:H. These two materials are both impurity rich and therefore inherently hinder the electronic transport. It is therefore reasonable to consider the charge impurity scattering as the dominant mechanism. Nevertheless, the temperature dependence of the phonon scattering often observed on graphene on $\mathrm{SiO}_{2}$ is not absent but rather postponed beyond $300 \mathrm{~K}$ as discussed by Chen et al. for impurity rich samples by [26,44]. Nevertheless, despite this limitation, the magnitude of the mobility observed in the capped graphene remains very interesting for the largescale application of graphene. As shown in Figure 4, the graphene films capped with p-type a-Si:H have a higher mobility and carrier concentration, leading to a square resistance of $420 \mathrm{Ohm} / \mathrm{square}$, which makes them suitable as an electrode for heterojunction solar cells and/or gate contact.

At last we have investigated the crystallization of a-Si:H on the Raman spectra using the $532 \mathrm{~nm}$ laser. The a-Si:H crystallizes at a power of $2.15 \mathrm{~mW}$. Figure 5 presents the Raman spectra for both heterostructures before and after crystallization. We observe that the a-Si:H peaks at $480 \mathrm{~cm}^{-1}$ and $2000 \mathrm{~cm}^{-1}$ disappear whereas the c-Si peak appears around $520 \mathrm{~cm}^{-1}$. 

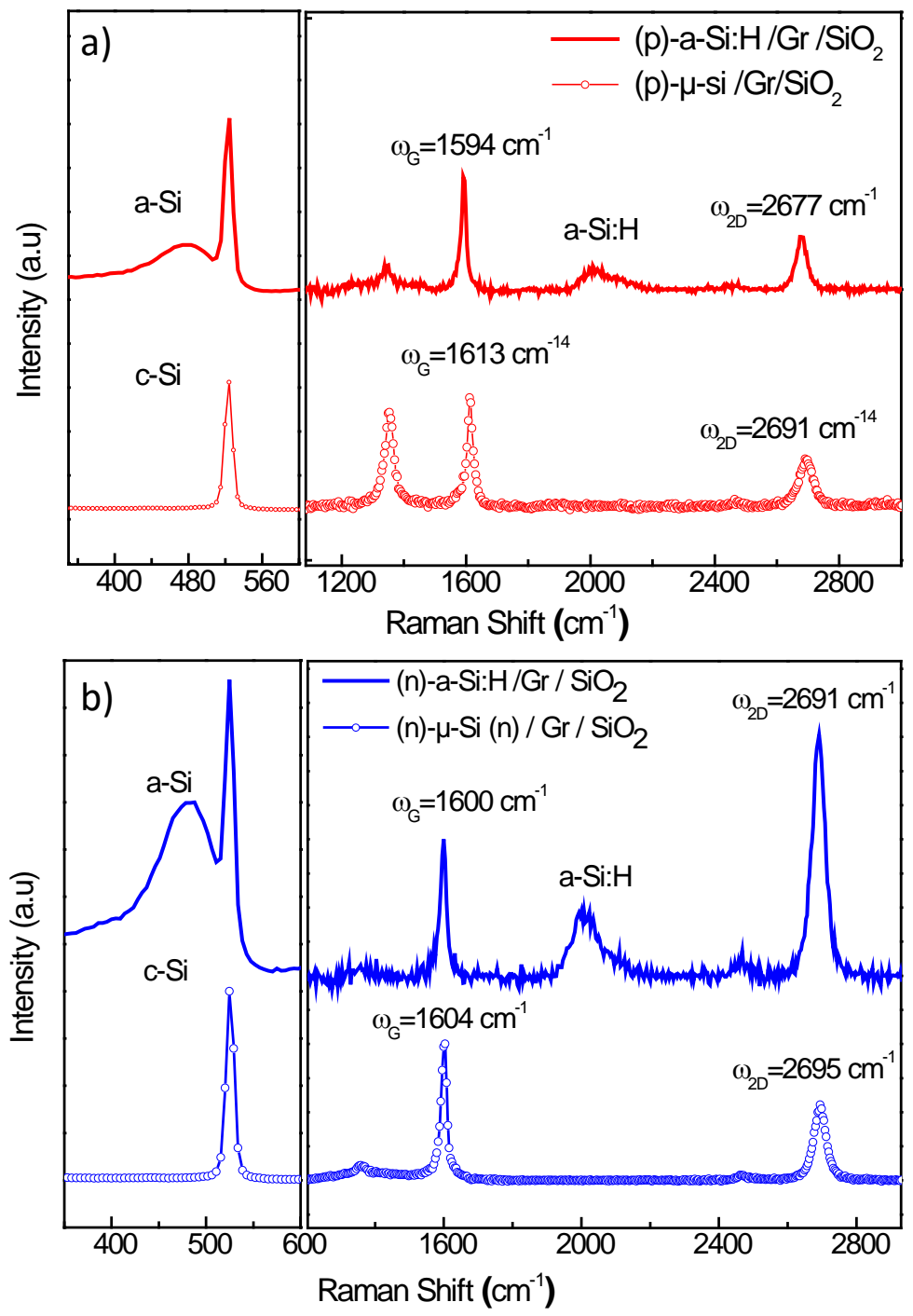

Figure 5: Raman spectra before and after crystallization of a) (p)-a-Si:H/Gr heterostructure and b) (n)-a-Si:H/Gr heterostructure. The as-deposited spectra present a shoulder at $480 \mathrm{~cm}^{-1}$ which is absent on the recrystallized spectra characterized by a single peak at $\sim 520 \mathrm{~cm}^{-1}$.

The $\mathrm{G}$ and 2D bands in the (p)-a-Si:H/Gr heterostructure exhibit an upshift of 19 and $14 \mathrm{~cm}^{-1}$ respectively. The (n)a-Si:H/Gr heterostructure, also presents upshifts for both $\mathrm{G}$ and 2D bands but of lower magnitude, $4 \mathrm{~cm}^{-1}$ for both $\mathrm{G}$ and 2D modes. This trend indicates two mechanisms: (i) the release of the compressive strain, (ii) a p-type doping. The increase in the D band intensity implies an increase in the defect density assigned to the volume change of the Si thin film during crystallization. It is particularly pronounced for the (p)-a-Si:H/Gr heterostructure. Moreover, the 2D/G ratio also reduces both samples suggesting an increase in carrier density i.e. doping originating from the $\mathrm{Gr} / \mathrm{SiO}_{2}$ and/or a-Si:H/Gr interfaces. The strength is nevertheless stronger for the (p)-a-Si:H/Gr heterostructure. It is known that (p)-a-Si:H conductivity increases after crystallization[45] and therefore more charges are available for transferring to the graphene. These data show that embedded CVD graphene maintains its features and the transport properties can be modulated according to the nature and the phase of the deposited a-Si:H. 


\section{Conclusion}

In this work, we have investigated the capping of CVD graphene film by n- and p-type doped a-Si:H. The deposition process of the a-Si:H has a limited impact on the structural integrity of graphene as observed by Raman spectroscopy and the graphene features are clearly visible on all heterosturctures. However the electronic properties are affected whether graphene is capped by n- or p-type a-Si:H. Post deposition Raman shifts indicate that the mechanical strain is dominating however the reduction of the $I_{2 D} / I_{G}$ ratio particularly for the (p)-a-Si:H/Gr heterostructure suggests a field effect doping as well. This observation has been evidenced by Hall effect measurements showing a substantial reduction of the sheet resistance. The transport is limited by the charge impurities from the $\mathrm{SiO}_{2}$ and the doped a-Si:H, the hole mobility reaches up to $1400 \mathrm{~cm}^{2} / \mathrm{Vs}$ without temperature dependence suggesting that phonon scattering is not dominant up to $300 \mathrm{~K}$ but would appear at higher temperature. At last we have shown that the recrystallization of the doped a-Si:H film relaxes the mechanical strain and only the

field effect doping remains. We have demonstrated that embedding CVD graphene under a-Si:H is a route for large scale graphene based solar cells or displays applications.

\section{ACKNOWLEDGMENT}

The authors would like to thank the Ministère des Affaires Etrangères et Européennes (MAEE) and CNRS for their financial support through the STIC ASIA programme (3226/DGM/ATT/RECH). This work was also supported by a public grant overseen by the French National Research Agency (ANR) as part of the "Investissements d’Avenir” program (Labex NanoSaclay, reference: ANR-10-LABX-0035). 


\section{REFERENCES}

[1] Maitra U, Matte H S S R, Kumar P and Rao C N R 2012 Strategies for the Synthesis of Graphene, Graphene Nanoribbons, Nanoscrolls and Related Materials Chim. Int. J. Chem. 66 941-8

[2] Kumar A and Huei C 2013 Synthesis and Biomedical Applications of Graphene: Present and Future Trends Advances in Graphene Science ed M Aliofkhazraei (InTech)

[3] Boutchich M, Arezki H, Alamarguy D, Ho K-I, Sediri H, Gunes, Fethullah F, Alvarez J, Kleider J, Lai C and Ouerghi A 2014 Atmospheric pressure route to epitaxial nitrogen-doped trilayer graphene on 4H-SiC (0001) substrate Appl. Phys. Lett. 105233111

[4] Ho K-I, Boutchich M, Su C-Y, Moreddu R, Marianathan E S R, Montes L and Lai C-S 2015 A Self-Aligned High-Mobility Graphene Transistor: Decoupling the Channel with Fluorographene to Reduce Scattering Adv. Mater. 27 6519-25

[5] Brus V V, Gluba M A, Zhang X, Hinrichs K, Rappich J and Nickel N H 2014 Stability of graphene-silicon heterostructure solar cells Phys. Status Solidi A 211 843-7

[6] Petridis C, Konios D, Stylianakis M M, Kakavelakis G, Sygletou M, Savva K, Tzourmpakis P, Krassas M, Vaenas N, Stratakis E and Kymakis E 2016 Solution processed reduced graphene oxide electrodes for organic photovoltaics Nanoscale Horiz

[7] Criado A, Melchionna M, Marchesan S and Prato M 2015 The Covalent Functionalization of Graphene on Substrates Angew. Chem. Int. Ed. 54 10734-50

[8] Bartolomeo A D, Giubileo F, Santandrea S, Romeo F, Citro R, Schroeder T and Lupina G 2011 Charge transfer and partial pinning at the contacts as the origin of a double dip in the transfer characteristics of graphene-based field-effect transistors Nanotechnology 22275702

[9] Bong J H, Sul O, Yoon A, Choi S-Y and Cho B J 2014 Facile graphene n-doping by wet chemical treatment for electronic applications Nanoscale 68503

[10] Loh K P, Tong S W and Wu J 2016 Graphene and Graphene-like Molecules: Prospects in Solar Cells J. Am. Chem. Soc. 138 1095-102

[11] Aktürk O Ü and Tomak M 2010 Bismuth doping of graphene Appl. Phys. Lett. 9681914

[12] Boutchich M, Arezki H, Alamarguy D, Ho K, Haikel S, Gunes F, Alvarez J, Kleider J-P, Lai C-S and Ouerghi A 2014 Electronic Properties of Large Area Nitrogen Doped Trilayer Graphene on 4H-SiC (0001)

[13] Dong X, Fu D, Fang W, Shi Y, Chen P and Li L-J 2009 Doping Single-Layer Graphene with Aromatic Molecules Small 5 1422-1426

[14] Stylianakis M M, Konios D, Kakavelakis G, Charalambidis G, Stratakis E, Coutsolelos A G, Kymakis E and Anastasiadis S H 2015 Efficient ternary organic photovoltaics incorporating a graphene-based porphyrin molecule as a universal electron cascade material Nanoscale 7 17827-35

[15] Stylianakis M M, Sygletou M, Savva K, Kakavelakis G, Kymakis E and Stratakis E 2015 Photochemical Synthesis of Solution-Processable Graphene Derivatives with Tunable Bandgaps for Organic Solar Cells Adv. Opt. Mater. 3 658-66

[16] Georgakilas V, Tiwari J N, Kemp K C, Perman J A, Bourlinos A B, Kim K S and Zboril R 2016 Noncovalent Functionalization of Graphene and Graphene Oxide for Energy Materials, Biosensing, Catalytic, and Biomedical Applications Chem. Rev. 116 5464-519 
[17] Kwon K C, Choi K S and Kim S Y 2012 Increased Work Function in Few-Layer Graphene Sheets via Metal Chloride Doping Adv. Funct. Mater. 22 4724-31

[18] Das S, Sudhagar P, Ito E, Lee D, Nagarajan S, Lee S Y, Kang Y S and Choi W 2012 Effect of HNO3 functionalization on large scale graphene for enhanced tri-iodide reduction in dye-sensitized solar cells $J$. Mater. Chem. 2220490

[19] Gunes F, Arezki H, Pierucci D, Alamarguy D, Alvarez J, Kleider J-P, Dappe Y J, Ouerghi A and Boutchich M 2015 Tuning the work function of monolayer graphene on 4H-SiC (0001) with nitric acid Nanotechnology 26445702

[20] Garg R, Dutta N K and Choudhury N R 2014 Work Function Engineering of Graphene Nanomaterials 4 267-300

[21] Kim B-J, Lee C, Mastro M A, Hite J K, Jr C R E, Ren F, Pearton S J and Kim J 2012 Buried graphene electrodes on GaN-based ultra-violet light-emitting diodes Appl. Phys. Lett. 10131108

[22] Yu X, Yang L, Lv Q, Xu M, Chen H and Yang D 2015 The enhanced efficiency of graphene-silicon solar cells by electric field doping Nanoscale 7 7072-7

[23] Petrone N, Dean C R, Meric I, van der Zande A M, Huang P Y, Wang L, Muller D, Shepard K L and Hone J 2012 Chemical Vapor Deposition-Derived Graphene with Electrical Performance of Exfoliated Graphene Nano Lett. 12 2751-6

[24] Gluba M A, Amkreutz D, Troppenz G V, Rappich J and Nickel N H 2013 Embedded graphene for large-area silicon-based devices Appl. Phys. Lett. 10373102

[25] Chauhan J and Guo J 2011 Inelastic Phonon Scattering in Graphene FETs IEEE Trans. Electron Devices 58 3997-4003

[26] Chen J-H, Jang C, Adam S, Fuhrer M S, Williams E D and Ishigami M 2008 Charged-impurity scattering in graphene Nat. Phys. 4 377-81

[27] Chen J-H, Jang C, Xiao S, Ishigami M and Fuhrer M S 2008 Intrinsic and extrinsic performance limits of graphene devices on SiO2 Nat. Nanotechnol. 3 206-9

[28] Yasaei P, Fathizadeh A, Hantehzadeh R, Majee A K, El-Ghandour A, Estrada D, Foster C, Aksamija Z, Khalili-Araghi F and Salehi-Khojin A 2015 Bimodal Phonon Scattering in Graphene Grain Boundaries Nano Lett. 15 4532-40

[29] Alexeev A M, Hartmann R R and Portnoi M E 2015 Two-phonon scattering in graphene in the quantum Hall regime Phys. Rev. B 92

[30] Ho P-H, Lee W-C, Liou Y-T, Chiu Y-P, Shih Y-S, Chen C-C, Su P-Y, Li M-K, Chen H-L, Liang C-T and Chen C-W 2015 Sunlight-activated graphene-heterostructure transparent cathodes: enabling high-performance n-graphene/p-Si Schottky junction photovoltaics Energy Env. Sci 8 2085-92

[31] Kiraly B, Mannix A J, Hersam M C and Guisinger N P 2015 Graphene-Silicon Heterostructures at the TwoDimensional Limit Chem. Mater. 27 6085-90

[32] Hong A J, Song E B, Yu H S, Allen M J, Kim J, Fowler J D, Wassei J K, Park Y, Wang Y, Zou J, Kaner R B, Weiller B H and Wang K L 2011 Graphene Flash Memory ACS Nano 5 7812-7

[33] Lee C, Wei X, Kysar J W and Hone J 2008 Measurement of the Elastic Properties and Intrinsic Strength of Monolayer Graphene Science 321 385-8 
[34] Lee G-H, Cooper R C, An S J, Lee S, van der Zande A, Petrone N, Hammerberg A G, Lee C, Crawford B, Oliver W, Kysar J W and Hone J 2013 High-Strength Chemical-Vapor-Deposited Graphene and Grain Boundaries Science 340 1073-6

[35] GÜNEŞ F, Han G H, Kim K K, Kim E S, Chae S J, Park M H, Jeong H-K, Lim S C and Lee Y H 2009 Large-area graphene-based flexible transparent conducting films Nano 4 83-90

[36] Wu Q, Jung S J, Jang S K, Lee J, Jeon I, Suh H, Kim Y H, Lee Y H, Lee S and Song Y J 2015 Controllable poly-crystalline bilayered and multilayered graphene film growth by reciprocal chemical vapor deposition Nanoscale 7 10357-61

[37] Roca i Cabarrocas P 1991 A fully automated hot-wall multiplasma-monochamber reactor for thin film deposition J. Vac. Sci. Technol. Vac. Surf. Films 92331

[38] Vignoli S, Mélinon P, Masenelli B, Cabarrocas P R i, Flank A M and Longeaud C 2005 Over-coordination and order in hydrogenated nanostructured silicon thin films: their influence on strain and electronic properties J. Phys. Condens. Matter 17 1279-88

[39] Cresti A, Lopez-Bezanilla A, Ordejón P and Roche S 2011 Oxygen Surface Functionalization of Graphene Nanoribbons for Transport Gap Engineering ACS Nano 5 9271-7

[40] Mohiuddin T, Lombardo A, Nair R, Bonetti A, Savini G, Jalil R, Bonini N, Basko D, Galiotis C, Marzari N, Novoselov K, Geim A and Ferrari A 2009 Uniaxial strain in graphene by Raman spectroscopy: G peak splitting, Grüneisen parameters, and sample orientation Phys. Rev. B 79

[41] Huang M, Yan H, Heinz T F and Hone J 2010 Probing Strain-Induced Electronic Structure Change in Graphene by Raman Spectroscopy Nano Lett. 10 4074-9

[42] Casiraghi C 2009 Probing disorder and charged impurities in graphene by Raman spectroscopy Phys. Status Solidi RRL - Rapid Res. Lett. 3 175-7

[43] Zabel J, Nair R R, Ott A, Georgiou T, Geim A K, Novoselov K S and Casiraghi C 2012 Raman Spectroscopy of Graphene and Bilayer under Biaxial Strain: Bubbles and Balloons Nano Lett. 12 617-21

[44] Fratini S and Guinea F 2008 Substrate-limited electron dynamics in graphene Phys. Rev. B 77

[45] Hamma S and Roca i Cabarrocas P 2001 Low-temperature growth of thick intrinsic and ultrathin phosphorous or boron-doped microcrystalline silicon films: Optimum crystalline fractions for solar cell applications Sol. Energy Mater. Sol. Cells 69 217-39 